\title{
Optimization of Cable Fault Recognition System Using Particle Swarm Optimization
}

\author{
Wong Jee Keen Raymond ${ }^{1}$, Chan Hou Jing ${ }^{2}$, Tze Mei Kuan ${ }^{3}$ \\ ${ }^{1}$ Dept. of Electrical \& Electronics Engineering, Tunku Abdul Rahman University College, Kuala Lumpur, \\ Malaysia,wongjk@tarc.edu.my \\ ${ }^{2}$ Dept. of Electrical \& Electronics Engineering, Tunku Abdul Rahman University College, Kuala Lumpur, \\ Malaysia, houjingchan@yahoo.com \\ ${ }^{3}$ Dept. of Electrical and Electronics Engineering, College of Engineering, Universiti Tenaga Nasional, Selangor, \\ Malaysia, tzemei@uniten.edu.my
}

\begin{abstract}
In this paper, a Partial Discharge (PD) based cable fault recognition system has been constructed using Artificial Neural Network (ANN), Support Vector Machine (SVM) and Adaptive Neuro Fuzzy Inference System (ANFIS). The cable fault recognition system can perform well under noise free condition but endures performance deterioration when PD noise contamination is present. Particle Swarm Optimization (PSO) was used to enhance the performance of classifiers under noise contamination. A performance review has been done to compare the optimized and unoptimized cable fault recognition under noise contamination. Results show that PSO optimized cable fault recognition systems perform better compared to unoptimized cable fault recognition systems. Among the optimized cable fault recognition systems, ANN outperforms SVM and ANFIS.
\end{abstract}

Key words: Cable, fault classification, partial discharge.

\section{INTRODUCTION}

The manifestation of PD in electrical equipment had been discovered as early as the start of 1990s [1]. PD is characterized as the discharge that does not entirelylink the areaamong the electrodes [2]-[3]. PD is intricately correlated to the breakdown of electrical insulation and is known to be the early hint of developing faults. Consequently, by observing PD activity to detect looming insulation failure, maintenance activity can be executed before catastrophic failure occurs [4]. Since PD is correlated to the defect type, a fault classification system can be developed by using machine learning techniques. Copious amount of research has been done on PD classification of numerous common power system equipment such as power transformer, power cable, substation and gas insulated switchgear [5]-[7]. When performed at a noise free environment, PD classification systems can achieve near perfect accuracy. However, actual PD classification in real-world situation is frequently hampered by noise contamination. Noise contamination is defined as PD that does not come from the test subject [8]. When clean PD data is covered with noise, the PD classification accuracy will decrease significantly.

PSO is a type of evolutionary computation methodsemployed for global optimization. PSO is motivated by the social behavior of the bird flocking. Due to its implementation simplicity, PSO is widely used to solve real-world problems [9]. PSO was used in [10] for localizing PD source using ultrasonic measurement in power transformer, PSO can converge quicker, perform calculations efficiently and avoidgetting trapped at the local optima when compare to Least-Square algorithm. A hybrid algorithm which combines PSO with neural network was proposed in [11] to identify PD acoustic signals, a variant of PSO known as Evolutionary PSO was used to determine the relevant parameter of the radial basis function network. Another study in [12] shows that PSO outperforms other traditional algorithms for PD localization in power transformer as it is able to avoid divergent problems. In this work, three machine learning methods, Artificial Neural Network (ANN), Support Vector Machine (SVM) and Adaptive Neuro Fuzzy Inference System (ANFIS) were used as cable fault classification system by classifying PD patterns. Naturally, all three classifiers will suffer performance degradation with the presence of the noise. Analtered version of Particle Swarm Optimization (PSO) known as Chaotic-Inertia Weight PSO with Time-varying Acceleration Coefficient (CIW-PSO-TVAC) was used to improve the parameters of the classifiers to enhance its capabilities under noise contamination.

\section{PD Data ACQuisition}

Five single core cable jointsof $11 \mathrm{kV}$ with artificial defects based on commonly observed defects in the field were made to generate five unique PD sources [13]. The defect type is tabulated in Table 1. The overallamount of 500 PD data was measured in this research where each defect contains 100 PD data and each PD data is of 1-minute duration The PD data is characterized in Phase-Resolved Partial Discharge (PRPD) pattern which has 3 dimensions, phase 
Jee Keen Raymond et al., International Journal of Emerging Trends in Engineering Research, 8(1.1), 2020, 147- 152

$(p h)$, charge $(Q)$, and intensity $(n)$. From the PRPD, two separate graphs can be derived which are the phase-charge graph $(p h-Q)$ and phase-intensity $(p h-n)$ graph. The graphs can be additionally split into positive half and negative halfcycles $(+v e-p h-Q, \quad-v e-p h-Q, \quad+v e-p h-n, \quad-v e-p h-n)$. Statistical features for example mean, standard deviation, skewness and kurtosis were calculated based on the PRPD graphs. Hence, a total of 16 input features as shown in Table 2 were extracted from the PRPD data to train the machine learning models.

Table 1: Common cable joint defect

\begin{tabular}{|c|l|}
\hline No. & \multicolumn{1}{|c|}{ Type of Defect } \\
\hline 1 & Insulation Incision \\
\hline 2 & Air Gap at Semiconductor Layer \\
\hline 3 & Semiconductor Layer Edge \\
\hline 4 & Axial Directional Shift \\
\hline 5 & Metal Particle on XLPE \\
\hline
\end{tabular}

Table 2: Extracted Input Features

\begin{tabular}{|c|l|c|l|}
\hline No. & \multicolumn{1}{|c|}{ Input feature } & No. & \multicolumn{1}{|c|}{ Input feature } \\
\hline 1 & Mean $(+v e-p h-Q)$ & 9 & Mean $(+v e-p h-n)$ \\
\hline 2 & Mean $(-v e-p h-Q)$ & 10 & Mean $(-v e-p h-n)$ \\
\hline 3 & Stdev $(+v e-p h-Q)$ & 11 & Stdev $(+v e-p h-n)$ \\
\hline 4 & Stdev $(-v e-p h-Q)$ & 12 & Stdev $(-v e-p h-n)$ \\
\hline 5 & Skewness $(+v e-p h-Q)$ & 13 & Skewness (+ve- $p h-n)$ \\
\hline 6 & Skewness $(-v e-p h-Q)$ & 14 & Skewness (-ve-ph-n) \\
\hline 7 & Kurtosis $(+v e-p h-Q)$ & 15 & Kurtosis (+ve-ph- $n)$ \\
\hline 8 & Kurtosis $(-v e-p h-Q)$ & 16 & Kurtosis (-ve-ph- $n)$ \\
\hline
\end{tabular}

\section{UnOPTIMIZEd Cable Fault ReCognition System}

Three types of machine learning were used as benchmark to observe the advantages gain by using the proposed optimization algorithm. Artificial neural network, support vector machine and adaptive neuro fuzzy inference system were used to classify PD patterns.

\subsection{Artificial Neural Network (ANN)}

A typical neural network was built usingMatlab utilizing the "patternnet" operation which is made up of 3 types of layers, the input layers, the hidden layers and the output layers. For this application, there are 16 neurons in the input layer, 10 neurons in the hidden layer and 5 neurons in the output layer. The ANN was trained using the back-propagation training algorithm. The ANN structure is depicted in Figure 1.

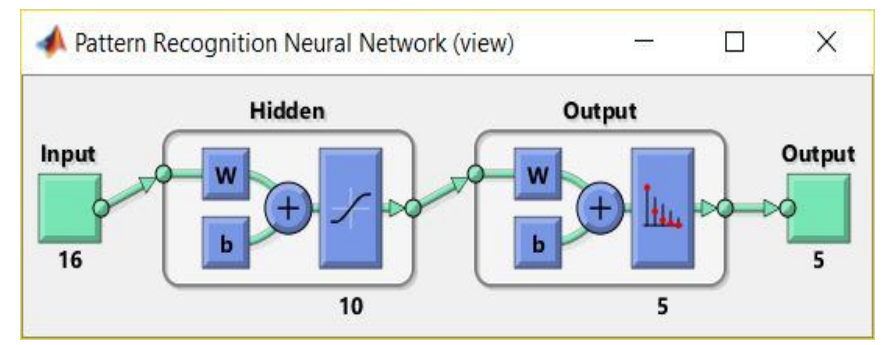

Figure 1: ANN structure

\subsection{Support Vector Machine (SVM)}

SVM is a type machine learning algorithm that is able to deal with the problems of complicated pattern recognition challenges. The earliest SVM algorithm is only capableof classify inputs into two category as it utilizes the hyperplane to divide the input data into two groups [14]. Therefore, a multi-level SVM [15] was used in this paper to recognize 5 types of PD defects. In Matlab, "svmclassify"was used to recognize the PD patterns after training using "svmtrain". Multilevel SVM was achieve by implementing SVM repeatedly. In the initial SVM classification, the entire inputs were categorized into Class 1 and not Class 1, the balance not Class 1 input were sent to the subsequent SVM to be grouped into Class 2 and not Class 2. The entire process was reiterated until just two classes are left over. The flow of this procedure is illustrated in Figure 2

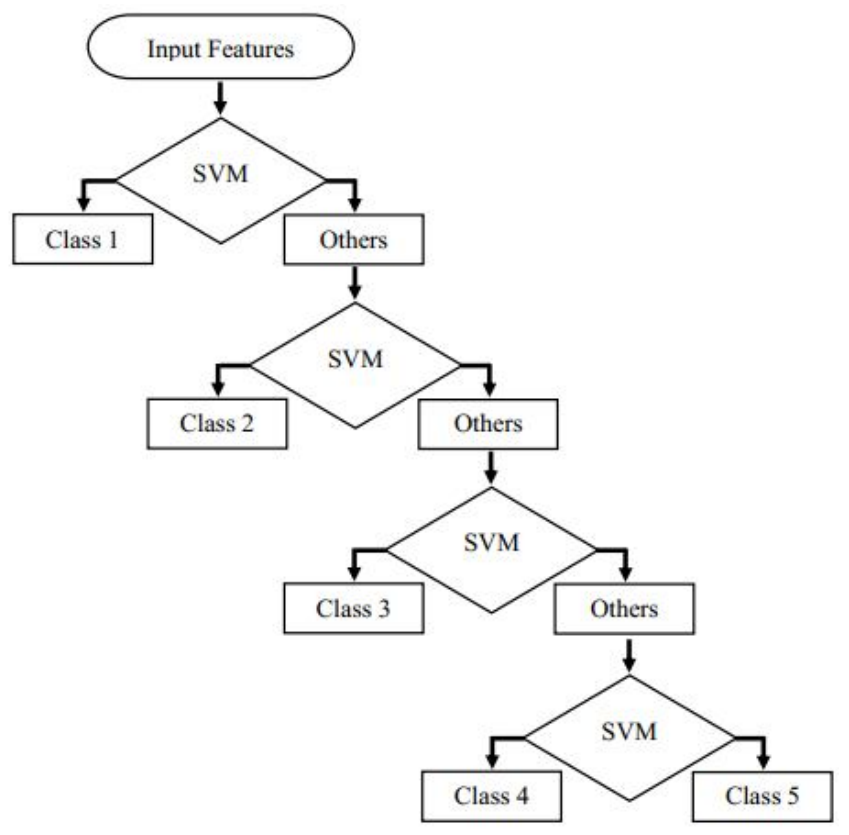

Figure 2: Multilevel SVM process

\subsection{Adaptive Neuro Fuzzy Inference System (ANFIS)}

ANFIS merge the philosophy of fuzzy inference system and artificial neural network to ascertain the bestsettingsof the PD classifiers. ANFIS possesses the ability to understand the relevant settings and adjusts the membership functions, therefore no precedingunderstanding of rule outcome and settings are needed to solve the question. It is not necessary to manually tune and set the settings of fuzzy system since the neural network will perform the selection automatically [16]. The "genfis2" command in Matlab was used to create a fuzzy inference system of Sugeno type that has subtractive clustering. The classifier was trained using the extracted input data to tune the fuzzy system's settings.

\section{MODIFIED PARTICLE SWARM OPTIMIZATION (PSO)}

Since the original PSO has a tendency to get trap at the local minima instead of convergingat the global best solution [17], 
analtered version of PSO known as CIW-PSO-TVAC was used in this work. Unlike the conventional PSO where the acceleration coefficients $\mathrm{c}_{1}, \mathrm{c}_{2}$ and inertia weight, $\mathrm{w}$ described in the particle velocity remain constant for all iterations as shown in (1) and (2), the variables of the modified version will changecorrelating to the iteration numbers.

$$
\begin{gathered}
v_{i j}(t+1)=w v_{i j}(t)+r_{1} c_{1}\left(p_{i j}(t)-x_{i j}(t)\right) \\
+r_{2} c_{2}\left(g_{j}(t)-x_{i j}(t)\right) \\
x_{i j}(t+1)=x_{i j}(t)+v_{i j}(t+1)
\end{gathered}
$$

For TVAC parameters, the maximum and minimum value range are adjusted to 2.5 and 0.5 correspondingly, and these values are considered as one of the most suitablerange of values for the optimization procedure as recommended by [18]. The TVAC parameters known as cognitive component $c_{1}$ and social coefficient, $c_{2}$ are calculated based on (3) and (4).

$$
\begin{aligned}
& c_{1}=\frac{\left(c_{1 f}-c_{1 i}\right) * I_{t}}{\text { MaxIt }}+c_{1 i} \\
& c_{2}=\frac{\left(c_{2 f}-c_{2 i}\right) * I_{t}}{\text { MaxIt }}+c_{2 i}
\end{aligned}
$$

$c_{l i}$ and $c_{l f}$ are the start value and end value for the cognitive component respectively whereas $c_{2 i}$ and $c_{2 f}$ are start value and end value for the social component respectively. ltand Maxltsignify the present iteration and highest iteration number of the PSO respectively. The rate of $c_{1}$ will decline from 2.5 down to 0.5 in order to permit the particles to travel the search space while avoiding it from converging too quickly toward the global best while the value of $c_{2}$ will rise from 0.5 up to 2.5 which increases the global search capability to guarantee that the result converges towards the global best while the amount of iteration increases. The nature of the acceleration coefficients is depicted in Figure 3.

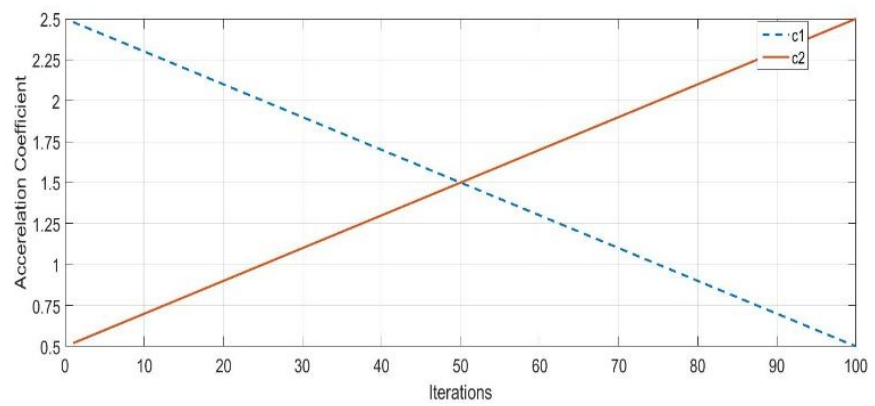

Figure 3: Acceleration Coefficients vs Iterations Numbers

The CIW is established on the linearlydecreasing inertia weight concept which combines with the chaotic movement to enhance the capability. Chaotic optimization has improved qualities in mountainclimbing and avoids the result from getting stuckat the local-minima [19].
To implement the notion of chaotic inertia weight, anarbitraryvaluezis first chosen in the range of $(0,1)$. Secondly, Logistic mapping, a type of the chaotic mapping methods had been selected in this paper using (5),

$$
z=4 * z *(1-z)
$$

After thezvalue had been calculated, it is applied into linearlydecreasing inertia weight equation as shown in 6 where $\mathrm{w}_{1}$ and $\mathrm{w}_{2}$ representing the starting weight as well as theending weight value.

$$
w=\left(w_{1}-w_{2}\right) * \frac{\text { MaxIt }-i t}{\text { MaxIt }}+\left(w_{2} * z\right)
$$

By utilizingthis sequence of measures, the inertia weight will contain chaotic characteristic while retaining its fluctuating trend. Figure 4 illustrates the linearlydecreasing inertia weight along with chaotic characteristic correlating to the increase in iterations.

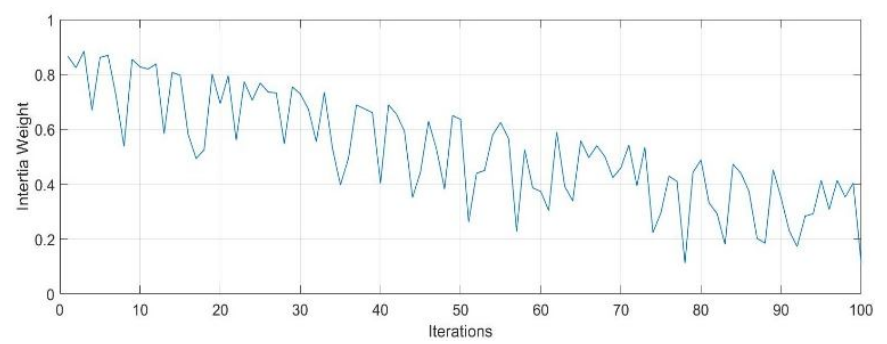

Figure4: LinearlydecreasingInertia Weight along with Chaotic Characteristic

After the calculated value was applied in the conventional PSO to substitute the constant inertia weight value, CIW-PSO-TVAC can then be implemented to improve the classifier. CIW-PSO-TVAC was employed to look for the initial weight connection of the ANN structure, then further training with local TRAINLM function, this methodology combine and utilize the ability of global search of PSO and local search of TRAINLM function in Matlab. For SVM, the CIW-PSO-TVAC was used to obtain the best pair of value for parameters of $\mathrm{C}$ and the Radial Basis Function sigma value for RBF kernel. For ANFIS, the number of the radii was improved by CIW-PSO-TVAC which consist of 17 values for 17 dimensions (1 output and 16 input feature) that indicate the center of cluster range of impact for every output and input data.

\section{ReSUlts AND ANALYSIS}

The performance of the cable fault classification system was tested under both noise free and noise contaminated situation which includes noise with rising pulse count as well as noise with rising charge magnitude. Noise with rising pulse count contains noisy signal lengths which ranges from 10 seconds up to 60 seconds with a 10 seconds step size. For the noise with rising pulse count, the noisy signal has an average charge magnitude ranging from $50 \mathrm{pC}$ to $250 \mathrm{pC}$ with a step size of 
Jee Keen Raymond et al., International Journal of Emerging Trends in Engineering Research, 8(1.1), 2020, 147- 152

$50 \mathrm{pC}$ each. The source of the noise signal was obtained from ground interference [20]. The PD classifiers ANN, SVM and ANFIS were be trained using noise free data and being benchmark by noise contaminated data to simulate real world performance.

\subsection{Noise Free Condition}

The performance of the cable fault classification system using all three machine learning algorithms is shown in Table 3. Under noise free condition, all three classifiers perform well with accuracy above $87.4 \%$. After being optimized by CIW-PSO-TVAC, ANN nearly achieves $100 \%$ accuracy under noise free condition. SVM and ANFIS also performed better with around $4 \%$ improvement after being optimized by CIW-PSO-TVAC.

Table 3: PD Classification Performance of Unoptimized vs Optimized Classifiers under Noise-Free Condition

\begin{tabular}{|c|c|c|}
\hline Classifiers & $\begin{array}{c}\text { Unoptimized Total } \\
\text { Accuracy }(\%)\end{array}$ & $\begin{array}{c}\text { Optimized Total } \\
\text { Accuracy }(\%)\end{array}$ \\
\hline ANN & 95.8 & 99.4 \\
\hline SVM & 90.6 & 94.8 \\
\hline ANFIS & 87.4 & 91.0 \\
\hline
\end{tabular}

\subsection{Noise with RisingPulse Count}

The effect of noise with rising pulse count on the classification accuracy is shown in Table 4. All 3 classifiers suffer a reduction in classification accuracy as the noise duration increases. However, after being optimized by CIW-PSO-TVAC, all 3 classifiers are able to retain a higher classification accuracy at all noise duration levels. At the maximum noise duration of 60 seconds, the classification accuracy of ANN increased from $82.4 \%$ to $90.4 \%$ while SVM increased from $69 \%$ to $80 \%$. The optimized ANFIS has better accuracy at every noise level except the maximum 60 seconds duration where it remains the same. Figure 5 to 7 shows the positive effect of using CIW-PSO-TVAC optimization for all three classifiers under noise with rising pulse count.

Table 4: PD Classification Performance of Unoptimized and Optimized Classifiers under Noise with rising Pulse Count

\begin{tabular}{|c|c|c|c|}
\hline \multirow{3}{*}{$\begin{array}{c}\text { Classif } \\
\text { iers }\end{array}$} & $\begin{array}{c}\text { Noise } \\
\text { Durati } \\
\text { on (s) }\end{array}$ & $\begin{array}{c}\text { Unoptimized } \\
\text { Total } \\
\text { Accuracy }(\%)\end{array}$ & $\begin{array}{c}\text { Optimized } \\
\text { Total } \\
\text { Accuracy (\%) }\end{array}$ \\
\hline \multirow{7}{*}{ ANN } & 0 & 95.8 & 99.4 \\
\cline { 2 - 4 } & 10 & 94.8 & 99.4 \\
\cline { 2 - 4 } & 20 & 93.2 & 99.2 \\
\cline { 2 - 4 } & 30 & 87.8 & 96.6 \\
\cline { 2 - 4 } & 40 & 86.4 & 93.2 \\
\cline { 2 - 4 } & 50 & 84.4 & 91.0 \\
\cline { 2 - 4 } & 60 & 82.4 & 90.4 \\
\hline \multirow{3}{*}{ SVM } & 0 & 90.6 & 94.8 \\
\cline { 2 - 4 } & 10 & 87.0 & 93.8 \\
\cline { 2 - 4 } & 20 & 83.0 & 90.6 \\
\hline
\end{tabular}

\begin{tabular}{|c|c|c|c|}
\hline \multirow{7}{*}{} & 30 & 77.2 & 88.0 \\
\cline { 2 - 4 } & 40 & 71.2 & 83.8 \\
\cline { 2 - 4 } & 50 & 68.4 & 80.6 \\
\cline { 2 - 4 } ANFIS & 60 & 69.0 & 80.0 \\
\hline \multirow{7}{*}{} & 0 & 87.4 & 91.0 \\
\cline { 2 - 4 } & 10 & 86.6 & 89.4 \\
\cline { 2 - 4 } & 20 & 84.2 & 88.0 \\
\cline { 2 - 4 } & 30 & 81.0 & 85.4 \\
\cline { 2 - 4 } & 40 & 79.2 & 81.4 \\
\cline { 2 - 4 } & 50 & 74.6 & 79.6 \\
\cline { 2 - 4 } & 60 & 73.6 & 73.6 \\
\hline
\end{tabular}

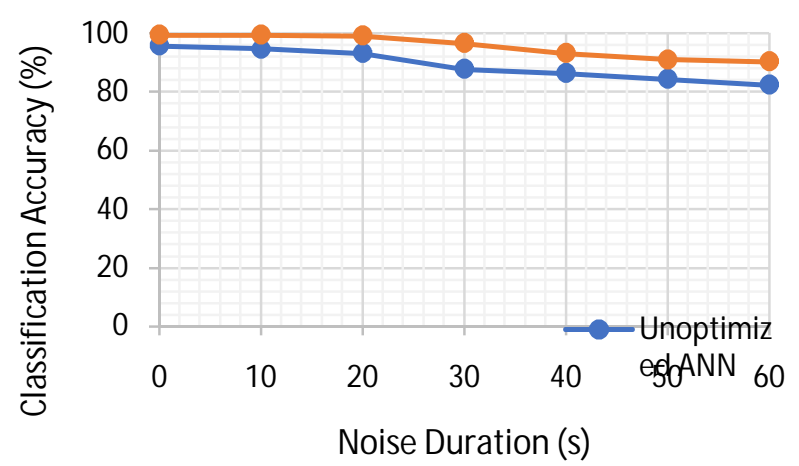

Figure 5: Effects of Rising Noise Duration on Unoptimized and Optimized ANN

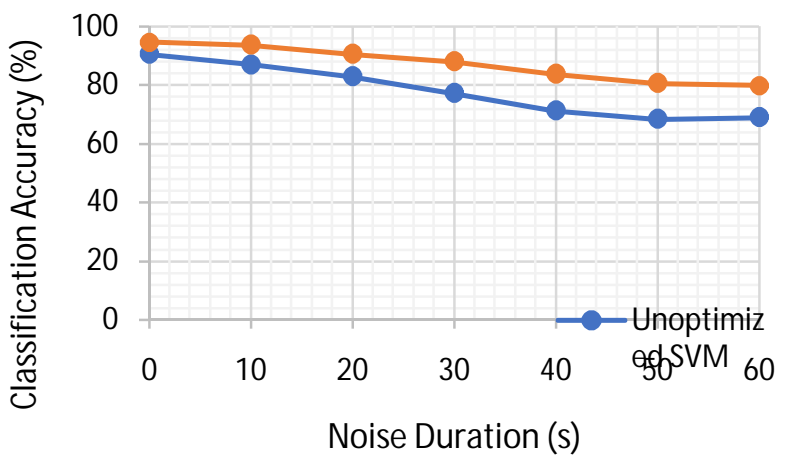

Figure 6: Effects of Rising Noise Duration on Unoptimized and Optimized SVM

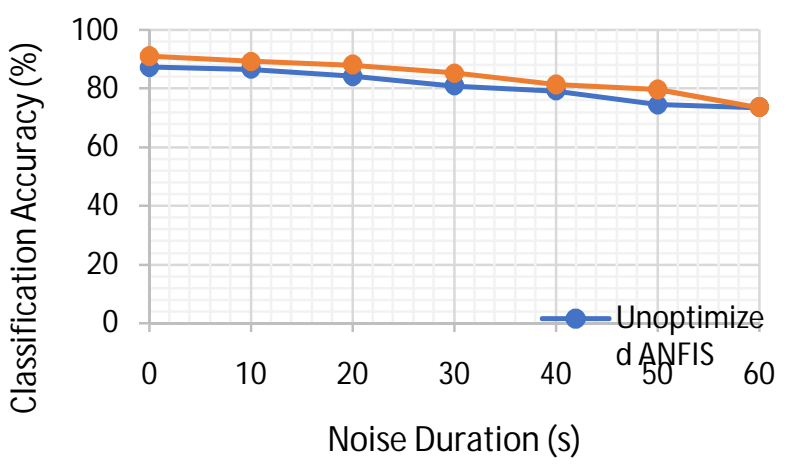

Figure 7: Effects of Rising Noise Duration on Unoptimized and Optimized ANFIS 
Jee Keen Raymond et al., International Journal of Emerging Trends in Engineering Research, 8(1.1), 2020, 147- 152

\subsection{Noise with Rising Charge Magnitude}

The effect of noise with rising charge magnitude on the classification accuracy is shown in Table 5. All 3 classifiers suffer a reduction in classification accuracy as the charge magnitude. The performance reduction due to noise with risingcharge magnitude is more severe compared to noise with risingpulse count. After being improved by CIW-PSO-TVAC, the classifiers were able to perform better than the unoptimized counterpart, but at a smaller margin. The improved ANN perform better when compare to unoptimized ANN under noise with risingcharge magnitude apart from under maximum noise level $250 \mathrm{pC}$ where it performed marginallyworst. Improved ANFIS performed better compared to unoptimized ANFIS at all charge magnitude levels with a $13 \%$ improvement at the maximum noise level. However, the overall classification accuracy is the weakest among the classifiers. For SVM, the optimized version performed worst after the noise level exceeds 200pC. Figure 8-10 shows the positive effect of using CIW-PSO-TVAC optimization for all three classifiers under noise with risingpulse count.

Table 5: PD Classification Performance of Unoptimized and Optimized Classifiers under Noise with Rising Charge Magnitude

\begin{tabular}{|c|c|c|c|}
\hline Classifiers & $\begin{array}{c}\text { Charge } \\
\text { Magnitude } \\
(\mathrm{pC})\end{array}$ & $\begin{array}{c}\text { Unoptimized } \\
\text { Total } \\
\text { Accuracy }(\%)\end{array}$ & $\begin{array}{c}\text { Optimized } \\
\text { Total } \\
\text { Accuracy } \\
(\%)\end{array}$ \\
\hline \multirow{5}{*}{ ANN } & 0 & 95.8 & 99.4 \\
\cline { 2 - 4 } & 50 & 94.4 & 99.2 \\
\cline { 2 - 4 } & 100 & 94 & 98 \\
\cline { 2 - 4 } & 150 & 91.2 & 96.2 \\
\cline { 2 - 4 } & 200 & 85 & 88.2 \\
\hline \multirow{5}{*}{ SVM } & 250 & 81.8 & 81.4 \\
\cline { 2 - 4 } & 0 & 90.6 & 94.8 \\
\cline { 2 - 4 } & 50 & 88.2 & 94 \\
\cline { 2 - 4 } & 100 & 86.4 & 90.4 \\
\cline { 2 - 4 } & 150 & 80.4 & 82.4 \\
\hline \multirow{5}{*}{ ANFIS } & 200 & 73.4 & 67.4 \\
\cline { 2 - 4 } & 250 & 68.4 & 54.8 \\
\cline { 2 - 4 } & 0 & 87.4 & 91 \\
\cline { 2 - 4 } & 50 & 85.6 & 89.8 \\
\cline { 2 - 4 } & 100 & 80.2 & 83.8 \\
\cline { 2 - 4 } & 150 & 68.6 & 73.2 \\
\hline & 200 & 59.4 & 65.6 \\
\hline
\end{tabular}

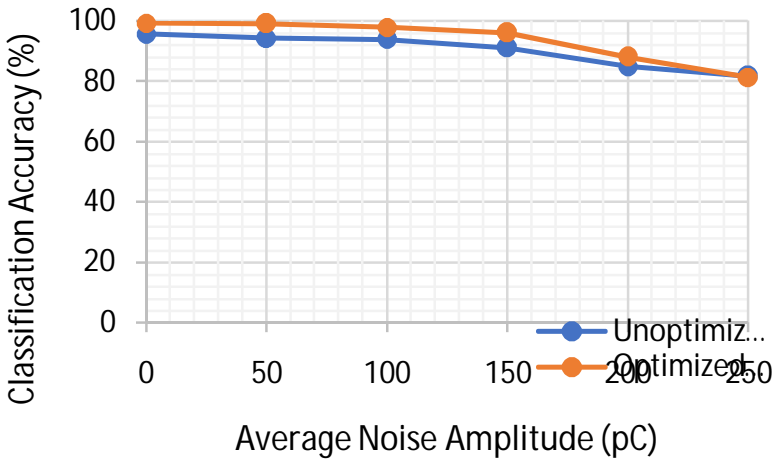

Figure 8: Effects of Rising Noise Amplitude on Unoptimized and Optimized ANN

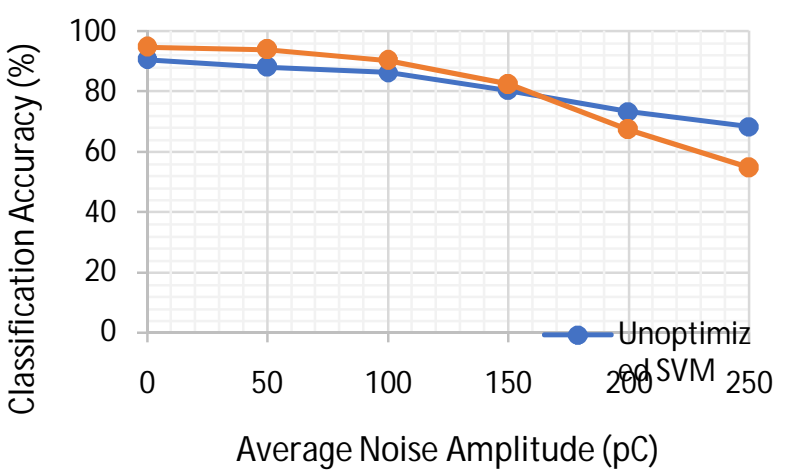

Figure 9: Effects of Rising Noise Amplitude on Unoptimized and Optimized SVM

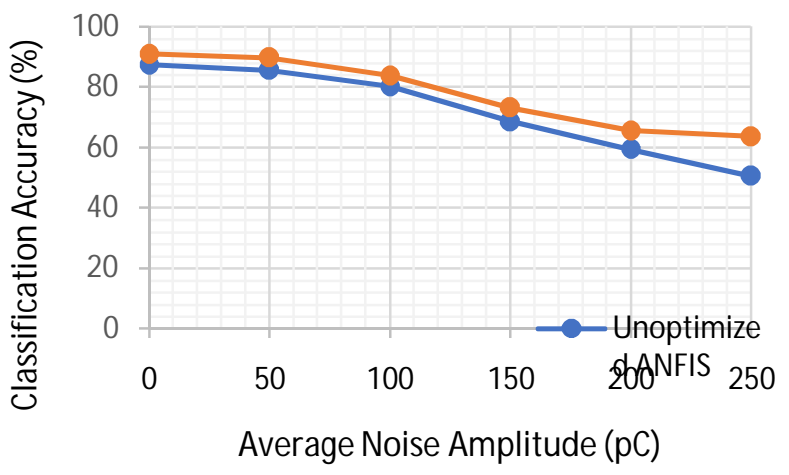

Figure 9: Effects of Rising Noise Amplitude on Unoptimized and Optimized ANFIS

\section{CONCLUSION}

In conclusion, the optimization of PD based cable fault classification system by utilizing CIW-PSO-TVAC was successfully performed. The classifiers tested include ANN, SVM and ANFIS were tested under noise free condition and two types of noise conditions which are noise with risingcharge magnitudeand noise with risingpulse count. With the CIW-PSO-TVAC optimization, all three classifiers are able to achieve higher tolerance to noise with risingpulse count. The effect of noise with risingcharge magnitude is more severe such that even with CIW-PSO-TVAC 
Jee Keen Raymond et al., International Journal of Emerging Trends in Engineering Research, 8(1.1), 2020, $147-152$

optimization, the noise tolerance improvement is marginal at the highest noise level. Overall, ANN is the best performer and most suited to be used as a cable fault classification system due to its higher noise tolerance level.

\section{ACKNOWLEDGEMENT}

This work would not be possible without the financial support of Tunku Abdul Rahman University College.

\section{REFERENCES}

1. A. Krivda, Automated recognition of partial discharges, IEEE Transactions on Dielectrics and Electrical Insulation, vol. 2, pp. 796-821, 1995.

2. 2. J. James, S. V. Kulkarni, and B. R. Parekh, Partial discharge in high voltage equipments-HV cable, in IEEE 9th International Conference on the Properties and Applications of Dielectric Materials, 2009, pp. 445-448.

3. W. J. K. Raymond, L. T. Sing, L. W. Kin, G. K. Meng, H. A. Illias, and A. H. A. Bakar, Feature Pruning for Partial Discharge Classification using IndFeat and ReliefF Algorithm, in IEEE 2nd International Conference on Dielectrics (ICD), 2018, pp. 1-4.

4. A. Aranburu, J. Olaizola, M. Barrenechea, P. Mulroy, A. Hurtado, and I. Gilbert, Signal processing techniques for on-line partial discharge detection and classification, in 24th European Signal Processing Conference (EUSIPCO), 2016, pp. 1433-1437.

5. L. Liping, T. Ju, and L. Yilu, Partial discharge recognition in gas insulated switchgear based on multi-information fusion, IEEE Transactions on Dielectrics and Electrical Insulation, vol. 22, pp. 1080-1087, 2015.

6. J. A. Hunter, P. L. Lewin, L. Hao, C. Walton, and M. Michel, Autonomous classification of PD sources within three-phase $11 \mathrm{kV}$ PILC cables, IEEE Transactions on Dielectrics and Electrical Insulation, vol. 20, pp. 2117-2124, 2013

7. W. Ke, L. Jinzhong, Z. Shuqi, L. Ruijin, W. Feifei, Y. Lijun, et al., A hybrid algorithm based on s transform and affinity propagation clustering for separation of two simultaneously artificial partial discharge sources, IEEE Transactions on Dielectrics and Electrical Insulation, vol. 22, pp. 1042-1060, 2015.

8. W. J. K. Raymond, H. A. Illias, A. H. A. Bakar, and H. Mokhlis, Partial discharge classifications: Review of recent progress, Measurement, vol. 68, pp. 164-181, 2015.

9. R. Poli, J. Kennedy, and T. Blackwell, Particle swarm optimization, Swarm Intelligence, vol. 1, pp. 33-57, June 012007.

10. T. Lijun, L. Richeng, D. Min, and S. Jun, Study of Partial Discharge Localization Using Ultrasonics in Power Transformer Based on Particle Swarm Optimization, IEEE Transactions on Dielectrics and Electrical Insulation, vol. 15, pp. 492-495, 2008.

11. N. A. Al-geelani, M. A. M. Piah, and N. Bashir, A review on hybrid wavelet regrouping particle swarm optimization neural networks for characterization of partial discharge acoustic signals, Renewable and Sustainable Energy Reviews, vol. 45, pp. 20-35, 2015/05/01/ 2015.

12. L. Ri-cheng, B. Kai, D. Chun, L. Shao-yu, and X. Guo-zheng, Study on Partial Discharge Localization by Ultrasonic Measuring in Power Transformer Based on Particle Swarm Optimization, in International Conference on High Voltage Engineering and Application, 2008, pp. 600-603.

13. W. Jee Keen Raymond, H. A. Illias, and A. H. Abu Bakar, Classification of Partial Discharge Measured under Different Levels of Noise Contamination, PLOS ONE, vol. 12, p. e0170111, 2017.

14. H. Guo and W. Wang, An active learning-based SVM multi-class classification model, Pattern Recognition, vol. 48, pp. 1577-1597, 2015/05/01/ 2015.

15. M. Carrasco, J. López, and S. Maldonado, A multi-class SVM approach based on the 11-norm minimization of the distances between the reduced convex hulls, Pattern Recognition, vol. 48, pp. 1598-1607, 2015/05/01/ 2015.

16. J. S. R. Jang, ANFIS: adaptive-network-based fuzzy inference system, IEEE Transactions on Systems, Man, and Cybernetics, vol. 23, pp. 665-685, 1993.

17. N. Lynn and P. N. Suganthan, Ensemble particle swarm optimizer, Applied Soft Computing, vol. 55, pp. 533-548, 2017/06/01/ 2017.

18. A. Ratnaweera, S. K. Halgamuge, and H. C. Watson, Self-organizing hierarchical particle swarm optimizer with time-varying acceleration coefficients, IEEE Transactions on Evolutionary Computation, vol. 8, pp. 240-255, 2004.

19. Y. Feng, G. F. Teng, A. X. Wang, and Y. M. Yao, Chaotic Inertia Weight in Particle Swarm Optimization, in Second International Conference on Innovative Computing, Informatio and Control (ICICIC), 2007, pp. 475-475.

20. W. J. K. Raymond, H. A. Illias, and A. H. A. Bakar, High noise tolerance feature extraction for partial discharge classification in XLPE cable joints, IEEE Transactions on Dielectrics and Electrical Insulation, vol. 24, pp. 66-74, 2017. 